\title{
EVALUACIÓN DE LOS EFECTOS DE LA ROTACIÓN EN OPERADORES DE MOTOSIERRAS ${ }^{1}$
}

\author{
Fidel Cándano Acosta ${ }^{2}$, Ariel Cuevas Bello ${ }^{3}$, Angelo Márcio Pinto Leite ${ }^{4}$, Carlos Cardoso Machado ${ }^{5}$, \\ Robson José de Oliveira ${ }^{6}$
}

\begin{abstract}
RESUMEN - El presente trabajo fue desarrollado en bosques naturales de Pinus caribaea, en la provincia de Pinar del Río-Cuba, para evaluar diferentes métodos en el corte raso con motosierra, empleando sistema de madera corta. Se experimentó la rotación del trabajo de un método de alternancia controlada de los operadores por el reabastecimiento de combustible de la motosierra (MAC) y un método con alternancia libre (MAL). En ambos métodos se utilizaron dos operadores en parejas, la palanca de empuje y la ejecución de la muesca direccional en árboles con diámetros superiores a $30 \mathrm{~cm}$. Los métodos fueron comparados entre si y a su vez, con el que se aplica tradicionalmente en estas áreas (T), que consta de un operador y un ayudante que mantienen sus funciones durante la jornada, no usan la palanca de empuje, no efectúan la muesca direccional y toman descanso espontáneos. De acuerdo con los resultados el MAC es el más apropiado pues, redujo los riesgos de accidentes y enfermedades ocupacionales en los operadores de motosierras, al equilibrar y disminuir la carga fisiológica de trabajo en un $13,69 \%$, redujo el tiempo de exposición a posturas incomodas, así como los efectos nocivos de la motosierra en un $52,08 \%$, la productividad del tiempo efectivo se elevó en un $27,32 \%$ respecto al método (T), con la disminución de los costos de la operación, que justifican salarios básicos correspondiente al nivel del operador para ambos integrantes de las parejas.
\end{abstract}

Palabras claves: Cosecha forestal, rotación del trabajo, carga fisiológica de trabajo.

\section{EVALUATION OF JOB ROTATION EFFECTS ON CHAINSAW OPERATORS}

\begin{abstract}
An study was carried out in the natural forests of Pinus caribaea, Pinar del Rio province, Cuba, aiming at the evaluation of the different methods used for clearcuting with chainsaw, using the cut-to-length system conducted in such a way to diminish the risks of the occupational diseases in chainsaw operators, and simultaneously to maintain the productivity levels and work quality. The rotation of the work was tested with an alternation controled method by refueling the chainsaw (MAC), and a free alternation method too (MAL). Both methods used: two chainsaw operators, the lever, and the directional felling in those trees exhibiting more than $40 \mathrm{~cm}$ diameter. These methods were compared to each other, and to that one traditionally applied on natural forests $(T)$, that consists of one operator and its assistant who besides maintaining their functions during this activity, do not use the lever nor make the directional felling, but rather adopt spontaneous pauses. According to the results, the MAC shows to be more appropriate because: it allows to reduce the risks of accidents and occupational diseases in chainsaw operators; provides the balance of the work physiologic load and the decreases of $13.69 \%$ in load and $52.08 \%$ in the noxious effects from chainsaw, although the productivity over the effective time showed an increase of $27.32 \%$ relative to method ( $T$ ), so diminishing the operating costs that justify the basic wages corresponding to the operator's level for both integrants of the team.
\end{abstract}

Key words: Cut of wood, work rotation, work physiologic load.

\footnotetext{
${ }^{1}$ Recebido para publicação em 12.11.2002 e aceito para publicação em 08.6.2004.

${ }^{2}$ Prof. Asistente del Departamento Forestal, Universidad de Pinar del Río, Cuba. E-mail: <candanoacosta@yahoo.es>.

${ }^{3}$ Doctor en Medicina General Especialista en Ciencia del Trabajo, Hospital Municipal "La Palma”. Pinar del Río.

${ }^{4}$ Prof. de las Faculdades Federais Integradas de Diamantina (FAFEID-DEF), Brasil. E-mail: <ampleite@ig.com.br>.

${ }^{5}$ Prof. de la Universidade Federal de Viçosa (UFV-DEF), Brasil. E-mail: <machado@ufv.br>.

${ }^{6}$ Ingeniero Forestal, MS, Universidade Federal de Viçosa (UFV), Brasil. E-mail: <engflorobson@ufv.br>.
} 


\section{INTRODUCCIÓN}

Dentro de las operaciones de cosecha de madera, el corte constituye la primera. De ahí que su eficiente ejecución repercuta directamente en las demás operaciones del proceso. La utilización de motosierra para el corte de madera en las empresas cubanas han sustituido, en gran escala, el uso de hachas y sierras manuales, debido a su alta productividad y la disminución de los desperdicios de madera.

A pesar de las ventajas productivas de las cosechadoras (Feller-bunchers), la motosierra continúa siendo una herramienta apropiada para las empresas forestales de Cuba, justificado por el bajo valor de inversión para su adquisición y manutención con respecto a máquinas más complejas. No obstante la motosierra es clasificada como una herramienta peligrosa (ROCHA Y SELL, 1989), él operador recibe el impacto directo de las vibraciones, el calor, los gases tóxicos y el ruido que se desprenden durante la ejecución de la labor. Para obtener la máxima eficiencia en la operación de corte con motosierra deben considerarse un grupo de factores ambientales, tecnológicos, organizativos, ergonómicos y humanos (SANT'ANNA, 1992; MINETTI, 1995 y MINETTI et al., 1999).

Los índices de accidentes entre los operadores de motosierras son elevados, aún cuando usan medios de protección personal. Los últimos datos informados por OIT (1981), los ubican en el primer lugar, con $91,4 \%$ de los accidentes ocurridos en la cosecha forestal. Sin embargo las enfermedades provocadas por la nocividad de la motosierra son el principal problema para estos trabajadores. Los obreros dedicados a esta labor pueden padecer: sordera, dedos blancos inducidos por vibración y afectaciones musculares, si no se toman medidas preventivas adecuadas.

Información obtenida de un grupo de operadores que realizan corte con motosierra con 4 años de experiencia en la empresa donde se efectúo esta investigación, indicó que el $43 \%$ presentan síntomas de las enfermedades más frecuentes provocadas por esta herramienta, tales como: dolores musculares $(50 \%)$, dolores lumbares y de espalda $(75 \%)$, hormigueo intermitente de los dedos (54\%), pérdida de sensibilidad de los dedos de las manos (51\%), dolores cervicales $(75 \%)$ y pérdida de la audición $(52 \%)$.
Considerando los antecedentes señalados, en este artículo se evalúa tres métodos de corte con motosierra, con la finalidad de disminuir los riesgos de accidentes y enfermedades ocupacionales en los operadores y mantener o incrementar la productividad en la operación.

\section{MATERIALES Y MÉTODOS}

Antes de realizar este trabajo, los operadores y ayudantes de motosierras fueron sometidos a una caracterización antropométrica y fisiológica. La edad promedio de los obreros fue de 37 años y el peso $67,4 \mathrm{~kg}$. La investigación incluyó a 10 operadores y 10 ayudantes, vinculados a la unidad silvícola San Andrés, municipio La Palma. El sistema de aprovechamiento fue el corte raso de árboles con motosierra Stihl 039.

\subsection{Descripción de los métodos de trabajo}

Método Tradicional (T): trabajo en pareja, un operador y un ayudante, sin alternancia en sus funciones y con prevalecía de pausas espontáneas.

Método de Alternancias Controladas (MAC): trabajo en pareja, ambos se comportan como operador y ayudante, alternando sus funciones al reabastecer de combustible la motosierra.

Método de Alternancias Libres (MAL): trabajo en pareja, ambos se comportan como operador y ayudante, alternando sus funciones libremente.

En el MAC y MAL se realizaron dos cortes en la cama o entalladura direccional, a árboles con diámetro superior a $30 \mathrm{~cm}$ y los ayudantes usaron palanca de empuje.

La duración de la jornada de trabajo fue de 8 horas, el 71,3\% del tiempo fue dedicado a la operación principal y el resto del tiempo se empleo en operaciones auxiliares y para el descanso de los obreros, 15 minutos por la mañana y 15 por la tarde. La jornada comenzaba a las 7:30 AM y terminaba a las 15:30 PM. Se realizaba un intervalo de 12:00 a 1:00 PM para el almuerzo.

El estudio se prolongó por 20 jornadas de trabajo. Durante toda la jornada se realizaron seguimientos telemétricos de la frecuencia cardiaca de los trabajadores por medio de un equipo digital adecuado para determinar la carga cardiovascular promedio. Se evaluaron otras variables tales como: el tiem- 
po de operación individual con la motosierra, los errores en la caída direccional de los árboles, la productividad y los costos de la operación.

Durante la jornada se evaluaron los tres métodos de trabajo de forma continua, realizando una fotografía del trabajo de cada pareja. Para lograr mayor uniformidad en el muestreo se controlaron las variables dasométricas, morfológicas, climáticas y del sujeto (nutrición). La intensidad de muestreo se calculó para un coeficiente de variación máximo del 10\%, un error de muestreo de 5\%. Se midio el diámetro y la longitud de los árboles y posteriormente se determino con la ayuda de una tabla de volumen elaborada por Grá et al. (1990), el volumen de los árboles.

\subsection{Características del área de trabajo}

El estudio se realizó en rodales naturales dePinus caribaea var. caribaea, donde el diámetro promedio de los árboles fue de $22,6 \mathrm{~cm}$, la altura de $14,8 \mathrm{~m}$, el volumen promedio de $0,25 \mathrm{~m}^{3} \mathrm{y}$ el volumen comercial por hectárea de $80 \mathrm{~m}^{3}$. Se tomaron observaciones en los meses de enero y febrero, estación más fría, y en julio y agosto estación de mayor temperatura. De acuerdo con Inrh (1996) en enero y febrero la temperatura media fue de $22,2^{\circ} \mathrm{C}$, la humedad relativa de $58,4 \%$ y las precipitaciones promedio de $665,6 \mathrm{~mm}$. En julio y agosto la temperatura media fue $28,7^{\circ} \mathrm{C}$, las precipitaciones de $1.046,2 \mathrm{~mm}$ y la humedad relativa de $80,3 \%$. El terreno es ondulado, con pendiente entre 12 y $25 \%$, ubicado a una altura de 127 metros sobre el nivel del mar. El suelo se clasifica como ferralítico (HERNÁNDEZ, 1994).

\section{RESULTADOS Y DISCUSIONES}

\subsection{Análisis del comportamiento del ritmo cardíaco en las parejas de obreros que trabajan con la motosierra}

Los efectos positivos de la rotación de funciones en los operadores de la motosierra se hacen evidentes al observar el comportamiento del ritmo cardíaco promedio durante cada jornada de trabajo (Figura 1).

Como se aprecia la aplicación del método tradicional propicio un desequilibrio entre la carga fisiológica de ambos obreros. La carga físiológica del operador es fuertemente moderada y el ayudante resulto ser baja según la clasificación dada por Sene (1994)

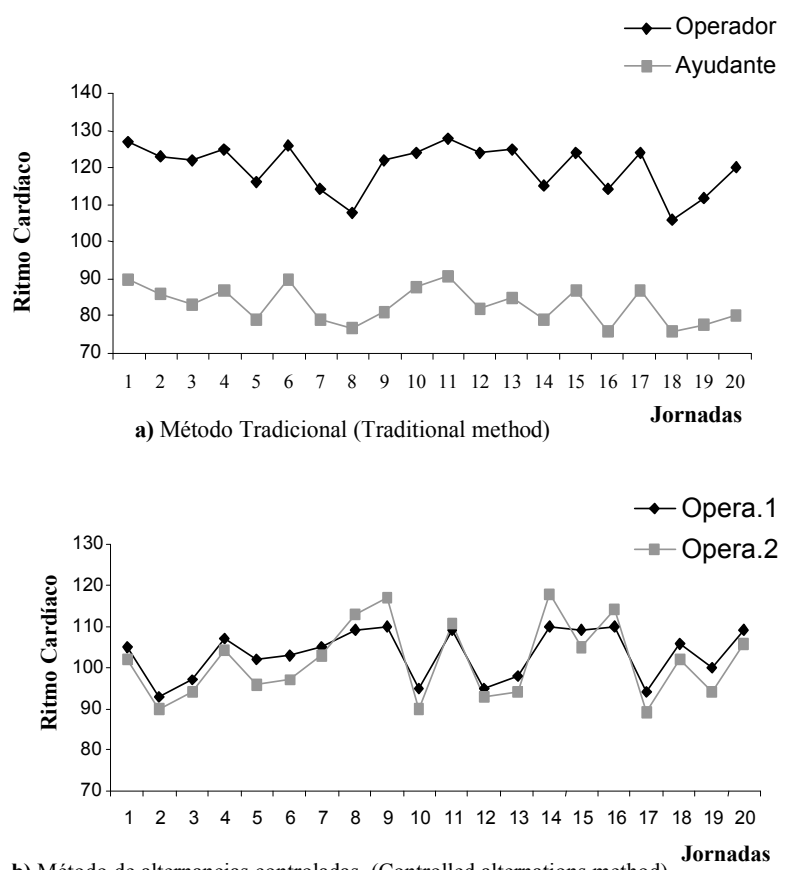

b) Método de alternancias controladas (Controlled alternations method)

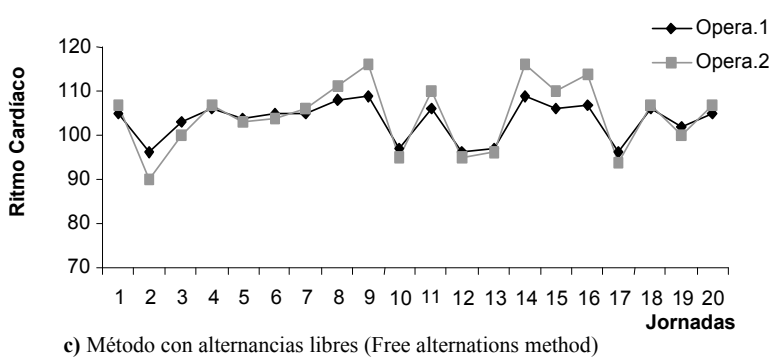

Figura 1 - Ritmo cardíaco versus jornada de trabajo. Figure 1 - Heart rhythm versus work day.

y Apud et al. (1995) para estas funciones. Sin embargo la carga fisiológica baja de los ayudantes no coincide con la reportada por los investigadores mencionados, los que determinaron cargas más elevada que la obtenida en esta investigación. La no correspondencia de estos resultados se explica por la diferencia en el contenido de trabajo de los ayudantes evaluados, que evidentemente es muy inferior. En los métodos 2 y 3 , se obtuvo una distribución más uniforme de la carga fisiológica, levemente moderada para ambos integrantes de la pareja, lo que evita mayores esfuerzos o mayor equilibrio en los esfuerzos. 
La figura 2 resume el comportamiento del ritmo cardíaco de los obreros durante las diferentes horas de la jornada de trabajo. Una tendencia general evidente es el incremento de la carga fisiológica con el aumento del tiempo de trabajo y la temperatura. También se aprecia que tanto para el método tradicional ( $\mathrm{T}$ ), como para el método con alternancias libres (MAL) en determinadas horas ocurre una alta carga fisiológica a diferencia del método con alternancias controladas (MAC), donde esta es uniforme y además para cualquier hora de la jornada la carga fisiológica no alcanzó valores tan altos que pudieran provocar daños a la salud de los obreros.
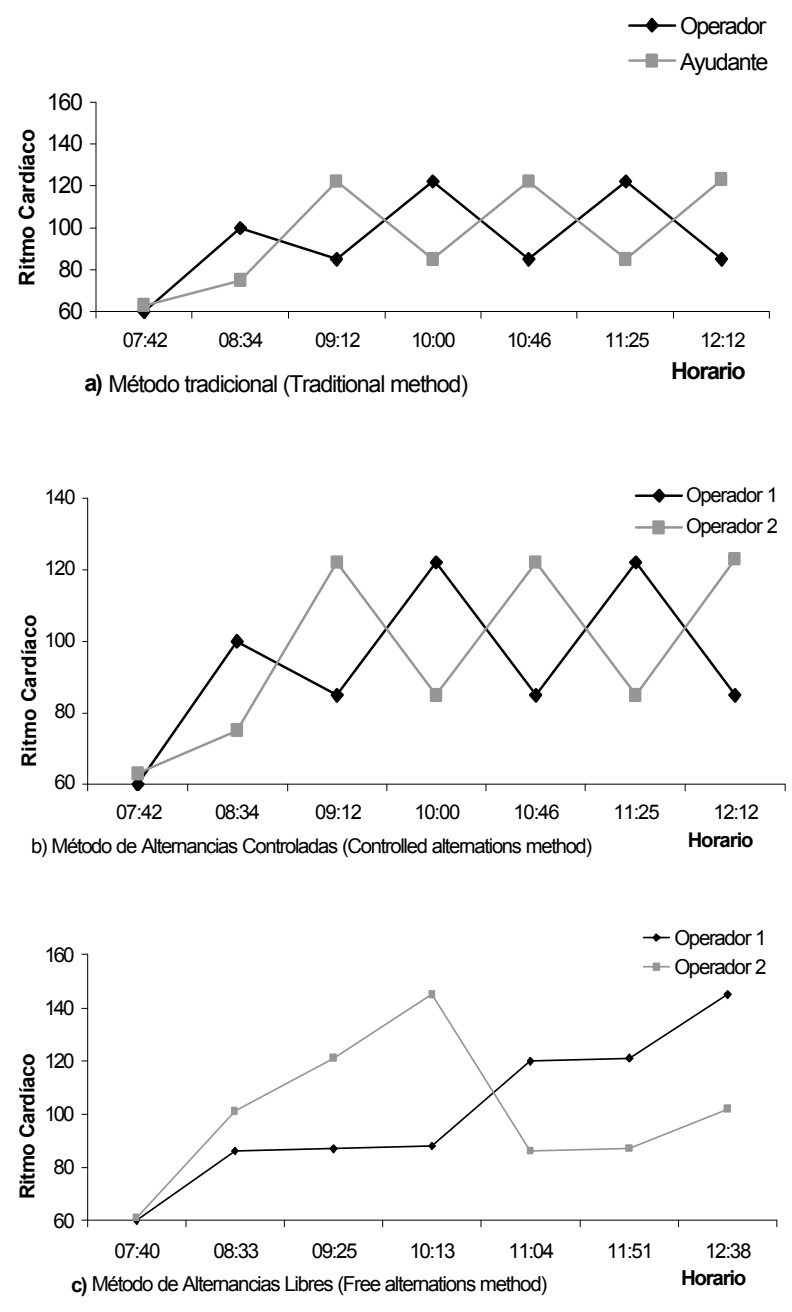

Figura 2 - Ritmo cardíaco versus horario. Figure 2 - Heart Rhythm versus Schedule.

\subsection{Análisis del tiempo promedio del trabajo con la motosierra para cada integrante de las parejas}

En la figura 3 se muestra la distribución del tiempo de trabajo efectivo de operación con la motosierra en cada integrante de la pareja con cada método de trabajo evaluado. En el método tradicional se observó un valor cero que corresponde al ayudante el cual no trabajó con la motosierra durante la jornada. El operador esta el 100\% del tiempo expuesto a los efectos negativos de la motosierra y a la vez realiza mayor esfuerzo físico.

En los métodos con alternancias (MAC y MAL) cada obrero trabajó un tiempo promedio muy similar durante la jornada, limitándose a la mitad del tiempo en comparación con el operador empleando el método tradicional. En el MAC se observa mejor distribución del tiempo de operación con la motosierra que el MAL, sin embargo lo significativo es que en este último método el número de rotación de las funciones de operador y ayudante fue muy baja ( 2 como promedio), y esto propició que la carga fisiológica de ambos obreros alcanzara valores más altos que los registrados por el MAC, con 4 rotaciones como promedio.

Los valores de productividad no difirieren entre el MAC y MAL (Figura 4), aunque es importante señalar que con la aplicación del MAL los obreros realizan mayores esfuerzos y aumentan los riesgos de accidentes en la operación con la motosierra con respecto al MAC. Se aprecia una diferencia significativa entre la productividad obtenida con el método tradicional (T) en relación a los métodos con alternancia. Esta

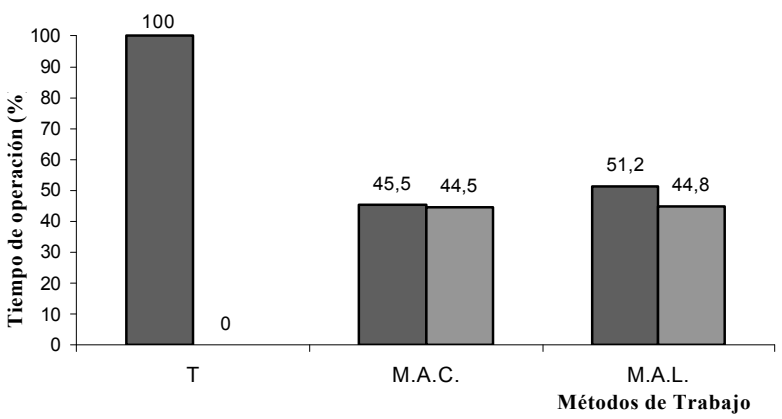

Figura 3 - Tiempo promedio de operación. Figure 3 - Average operational time. 
diferencia es más evidente al observar que la aplicación del método tradicional logró un $65.3 \%$ de utilización del tiempo productivo de la jornada de trabajo, contra $85.2 \%$ y $86.1 \%$ cuando se emplearon MAC y MAL, respectivamente.

\subsection{Análisis de la uniformidad de caída de los árboles}

La Figura 5 permite observar como la aplicación de MAC y MAL logra una disminución significativa de los errores en la caída de los árboles en comparación con el método tradicional. Se explica por el uso de la palanca de empuje y la ejecución de la entalladura direccional técnicamente superior y el adiestramiento recibido por los obreros sobre esta operación.

La mayor uniformidad de caída de los árboles facilitó la operación de extracción, al reducir el tiempo de amarre de la madera, con una sensible reducción de los costos $\mathrm{y}$, simultáneamente, redujo los daños en el arrastre de los fustes sobre la cubierta del suelo.

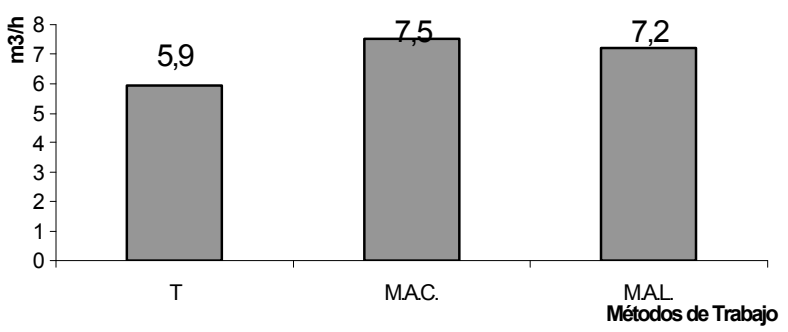

Figura 4 - Productividad Promedio del Equipo de Trabajo. Figure 4 -Average of the productivity per team work.

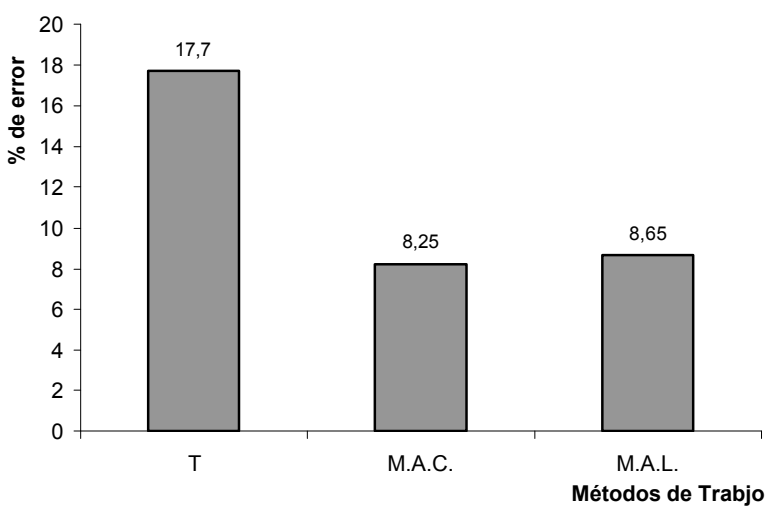

Figura 5 - Errores en la caída direccional de los árboles. Figure 5 -Errors in the directional fall of the trees.

\section{CONSIDERACIONES FINALES}

Después de evaluar los de tres métodos de trabajo con motosierra y los aspectos ergonómicos, productivos y económicos, se puede aseverar que el método de alternancia controlada (MAC) es la mejor opción para las condiciones donde se realizó la investigación debido a:

Se logra la reducción de la carga fisiológica de los obreros, en comparación con los resultados obtenidos por el método tradicional y el método de alternancia libre, lo cual implica disminución de las posibilidades de fatiga fisiológica por sobrecarga excesiva de trabajo y de riesgos de accidentes y enfermedades ocupacionales. Además se aumenta la productividad del trabajo y mayor uniformidad de caída de los árboles, que causa la reducción de los costos y de los daños a la cubierta vegetal en la operación de arrastre.

\section{REFERÊNCIAS BIBLIOGRÁFICAS}

APUD, E.A. Model to estimate reference yields in harvesting using ergonomically principles. In: SEMINÁRIO DE ATUALIZAÇÃO EM SISTEMAS DE COLHEITA DE MADEIRA E TRANSPORTE FLORESTAL, 8., 1994, Curitiba. Anais... Curitiba: UFPR/FUPEF, 1994. p. 23-31.

GRÁ, H. et al. Tabla dasométrica de Pinus caribaea. La Habana: Instituto de Investigaciones Forestales, 1990. 28 p. (Informe técnico).

HERNÁNDEZ, A. et al. Nueva versión de clasificación genética de los suelos de Cuba. Cuba: Ministerio de la Agricultura, La Habana, 1994.

INSTITUTO NACIONAL DE RECURSOS HIDRÁULICOS (I.N.R.H). Informe sobre registros climáticos. PR 159. La Pimienta y PR 434. La Amistad; San Andrés; La Palma; Pinar del Río, 1996. 11 p.

MinetTi, L.J. Análise de fatores operacionais e ergonômicos no corte florestal com motosserra. 1995. 221 f. Tese (Doutorado em Ciência Florestal) - Universidade Federal de Viçosa, Viçosa, 1995.

MinetTi, L.J. et al. Perfil do operador de motosserra profissional e do futuro do Brasil diante das questões cultural, política, regional e de competitividade. Viçosa: MG: UFV, 1999.p. 304-321.

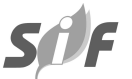

R. Árvore, Viçosa-MG, v.28, n.3, p. 367-372, 2004 
NORMA CUBANA 34-38: Máquinas

agropecuarias $y$ forestales: metodología para la evaluación económica. La Habana: 1985. 8 p.

OIT. Operational efficiency, work study and ergonomics in forestry. Ginebra: The United Republic of Tanzania, 1981.89 p.

ROCHA, A.M.; SELL, I.O. O reprojeto de uma motosserra. In: SEMINÁRIO BRASILEIRODE ERGONOMIA, 1989. Rio de Janeiro. Anais... Rio de Janeiro: 1989, p.160-165.
SANT'ANNA, C. M. Fatores relacionados com a produtividade do operador de motosserra. 1992. 145 f. Dissertação (Mestrado em Ciência Florestal) - Universidade Federal de Viçosa, Viçosa, 1992.

SENE, J. Análise fisiológica e biomecânica da função motosserrista. In: SEMINÁRIO DE ATUALIZAÇÃO EM SISTEMAS DE COLHEITA DE MADEIRAE TRANSPORTE FLORESTAL, 8., 1994, Curitiba. Anais... Curitiba: UFPR/FUPEF, 1994. p. 1-20. 